\title{
INTEGRAÇÃO DA GESTÃO DE PROJETOS E PROCESSSOS PARA IMPLEMENTAÇÃO DE UM ERP
}

\author{
${ }^{* 1}$ Nathan Peixoto Oliveira, ${ }^{2}$ Bruno da Silva Guerra, ${ }^{3}$ Rômulo Henrique Gomes de Jesus, \\ ${ }^{4}$ Danielle Gonçalves de Oliveira Prado \\ 1,2 Université de Bordeaux, 33000, Bordéus-AQ, França \\ ${ }^{3}$ Universidade Tecnológica Federal do Paraná, 84016-210, Ponta Grossa-PR, Brasil \\ ${ }^{4}$ Universidade Tecnológica Federal do Paraná, 86812-460, Apucarana- PR, Brasil \\ *nathanpeixot@yahoo.com.br
}

\begin{abstract}
RESUMO
A gestão organizacional integrada auxilia o alcance dos objetivos estratégicos da empresa, agregando valor aos clientes e criando vantagem competitiva. Deste modo, esse estudo visa apresentar uma abordagem para estruturação das interfaces entre projetos e processos em uma organização. Foi realizada uma pesquisa empírica com resultados interpretados à luz dos materiais teóricos e experimentais levantados na revisão bibliográfica. Assim, foram apontadas as principais diferenças entre os dois conceitos na implementação de um ERP. Esta visão integrada foi base para a identificação de iniciativas de melhoria, definição de critérios de prioridade e maximização das probabilidades de sucesso, além de dar suporte a gestão e a visão de longo prazo da empresa.
\end{abstract}

Palavras-chave: Gestão de Projetos; Gestão de Processos; ERP.

\section{INTEGRATION OF PROJECT AND PROCESS MANAGEMENT FOR ERP IMPLEMENTATION}

\begin{abstract}
Integrated organizational management helps achieve the company's strategic objectives, adding value to customers and creating competitive advantage. Thus, this study aims to present an approach for structuring the interfaces between projects and processes in an organization. An empirical research was performed with results interpreted in the light of the theoretical and experimental materials raised in the literature review. Thus, the main differences between the two concepts in the implementation of an ERP were pointed out. This integrated vision was the basis for identifying improvement initiatives, defining priority criteria and maximizing the likelihood of success, as well as supporting the company's management and long-term vision.
\end{abstract}

Keywords: Project Management; Process Management; ERP. 


\section{Como Citar:}

OLIVEIRA, Nathan Peixoto et al. Integração da gestão de projetos e processos para implementação de um ERP. In: SIMPÓSIO DE PESQUISA OPERACIONAL E LOGÍSTICA DA MARINHA, 19., 2019, Rio de Janeiro, RJ. Anais [...]. Rio de Janeiro: Centro de Análises de Sistemas Navais, 2019.

\section{INTRODUÇÃO}

\subsection{Apresentação do Tema}

Segundo Beinhocker (2007) toda vantagem competitiva é temporária. Anualmente as empresas tem o desafio de realizar o seu planejamento de curto, médio e longo prazo tendo como objetivo equilibrar esses três alcances de tempos, porém priorizando o planejamento de longo prazo visando atingir os melhores resultados para a empresa. Nessa rotina de planejamentos periódicos, muitas estratégias são sugeridas para alcançar os objetivos com aumento do faturamento, redução dos custos e fidelização de clientes, entre outros pontos necessários para o crescimento sustentável e seguro da organização, gerando mais competitividade e diferenciação para os negócios da empresa.

Uma empresa que deseje alcançar resultados de longo prazo, não deve focar apenas na excelência operacional, mas estabelecer como estratégia de negócios um modelo pautado na busca constante pela competitividade de forma diferenciada, sendo reciclada de tempo em tempo, conforme sua necessidade. Com todo esse cenário, a utilização de ferramentas que auxiliem na manutenção da vantagem competitiva será essencial para a sobrevivência e rentabilidade da organização.

Face a globalização, competitividade da concorrência e desejo crescente por rapidez em resultados, é cada vez maior a pressão por uma excelência em projetos e processos, cuja interação quando bem-sucedida, definem que as expectativas dos stakeholders foram atendidas e permanecerão em um ciclo de melhoria contínua. São, portanto, consideradas projetos de melhoria interna que ao seu término, se tornam processos de rotina da empresa.

Nesse contexto, surge a necessidade dessa pesquisa, para analisar e pautar melhorias advindas da relação entre a gestão de processos, gestão de projetos, seus alinhamentos e benefícios.

\subsection{OBJETIVo}

Demonstrar que a conduzir um bom relacionamento entre a Gestão de Projetos e Gestão de Processos, ligando da melhor forma essas duas ferramentas é o melhor caminho para se alcançar o máximo de resultados na implantação de um ERP. Além disso, de forma mais específica, buscou-se ressaltar a importância da Gestão da Mudança dentro do contexto da implantação de um ERP e o seu papel na interface entre a Gestão de Projetos e Gestão de Processos. Isto feito de forma clara e com benefícios práticos e comprovados nas empresas através das ferramentas propostas por esse estudo.

\subsection{JUSTIFICATIVA}

Transformações constantes na sociedade (culturais, tecnológicos, políticos, econômicos, sociais, entre outros) estão acontecendo com uma rapidez crescente. Num âmbito geral, é comum associarmos as mudanças significativas ao resultado de projetos (Vieira, 2002).

A gestão de projeto traz resultados temporários e inovadores para a empresa, enquanto o processo mantém a melhoria gerada por projetos bem executados. Segundo o PMI - Project Management Institute (2013) o planejamento é o fator mais importante para o sucesso de um projeto enquanto a ABMP - Association of Business Management Process 
(2013), ressalta que o fator preponderante para atingir resultados na gestão de processos é a eficácia na execução.

Entender a relação entre essas duas ferramentas de gestão é essencial para alcançar diferencial competitivo e realizar uma implantação de um ERP eficiente e que gere resultados a longo prazo para a empresa. Pois, a implantação de um ERP é realizada através da gestão de um projeto e essa implantação gera mudanças nos processos da empresa.

\subsection{Metodologia}

O primeiro passo da pesquisa foi uma revisão bibliográfica na temática de gestão de projeto e processos e seus alinhamentos para resultados práticos na implementação de ERPs, onde, para a realização dessa fase, foram consultados artigos e livros na temática. A seguir, foi feita uma pesquisa empírica com resultados interpretados à luz dos materiais teóricos e experimentais levantados, obtendo-se conclusões embasadas.

\section{FUNDAMENTAÇÃO TEÓRICA}

\subsection{Gestão de Projetos e Processos}

Um passo imprescindível para realização de uma tarefa de uma maneira mais eficiente e padronizada com o objetivo de reduzir erros e aumentar as chances de acertos é a utilização de uma ferramenta gerencial. No caso da Gestão de Projetos, é aquela que administra recursos limitados, prazos definidos, além de requisitos técnicos específicos. Inserido nesses desafios, surgiu o PMI - Project Management Institute, com o objetivo de identificar as melhores práticas no gerenciamento de projetos através da publicação do documento denominado "Project Management Body of Knowledge - PMBOK".

O guia PMI, (2013, p. 8) destaca que "o gerenciamento de projetos é a aplicação de conhecimento, habilidades, ferramentas e técnicas às atividades do projeto a fim de atender aos seus requisitos”. Esta tarefa é realizada por meio de processos que recebem entradas e geram saídas.

Os autores Moroni e Hansen (2006) descrevem de forma sintética as cinco etapas de um projeto como:

a) Iniciação: fase em que são feitas apresentações do projeto, definições do escopo, autorizações entre outros.

b) Planejamento: fase em que é feito um refinamento do projeto com a definição das atividades, alocação dos recursos, estimativas de custos e prazos, além da determinação de alternativas para atendimento dos objetivos.

c) Execução e Controle: fase em que o projeto é posto em prática e também quando são estabelecidos os princípios para controle dos recursos e prazos.

d) Encerramento: fase em que são criados documentos, relatórios de encerramento, além de ser o momento de se avaliar os pontos positivos e negativos como forma de auxiliar no aprendizado da organização.

Os projetos ou ainda mudanças são entendidos nas empresas como um requisito básico para garantia de crescimento do negócio. Este crescimento deve estar pautado pela consciência de preservação ambiental, pela garantia de segurança das pessoas e pela proteção dos bens materiais com o objetivo de gerar valor para a sociedade.

Conforme destaca Gasnier (2000), dentre as principais vantagens destas ferramentas estão o suporte à produtividade, comunicação, integração, simulação e mais eficácia nos controles. De uma forma geral, são aliados do gerente de projetos na busca pelo alinhamento entre o planejamento e a execução.

Já a gestão por processos, ou Business Process Management (BPM), é uma disciplina de gestão que exige das organizações uma mudança para o pensamento centrado 
nos processos (Doebeli et al., 2011). A importância de melhorar a organização de uma maneira eficiente e a necessidade de responder às exigências de mudanças endógenas e exógenas às organizações têm sido os maiores desafios contemporâneos (Ulmer et al., 2011). Por isso o BPM é apresentado como uma maneira de auxiliar as organizações a se adaptarem às necessidades de mudança. (PSOMAS et al., 2011). O BPM exige pessoas para tomar decisões sobre questões complexas que envolvem o uso de recursos tangíveis e intangíveis (CLEGG; SHAW, 2008).

A ideia central por trás do BPM é que as organizações devem coordenar os esforços de seus processos e gerencia-los como um ativo (HARMON; WOLF, 2012). Desta forma, o BPM tem recebido atenção significativa do mercado (DOEBELI et al., 2011).

Gestão de processos é um conceito antigo, sendo abordado de diversas formas por pesquisas e estudiosos em negócios e gestão de empresas. Durante 1970, os métodos para trabalhar com processos foram desenvolvidos sob os rótulos de just in time e produção enxuta por Schonberger (1986). Nos anos 1980, o Sistema Toyota de Produção lançou um novo olhar de desempenho e performance operacional no mundo. Dentre as características deste novo método de gestão se encontrava a melhoria sistemática dos processos para a obtenção contínua de resultados.

Nas décadas de 1980 e 1990, o escopo de trabalho do controle e gestão de processo foi aumentado para atuar o foco corporativo da época incluindo todas as áreas da organização. Neste período, uma grande parte da atenção foi voltada para a reengenharia de processos empresariais (HAMMER; CHAMPY, 1993). Desde 1980, gestão de processos tem sido descrito como a ideia mais importante dentre os modelos de gestão (HELLSTRÖM, 2006).

A reengenharia de processos foi sinônimo do renascimento do interesse na gestão de processos (HAMMER; CHAMPY, 1993). Entretanto, o resultado de várias formas de abordagens deste modelo nas empresas resultou em cortes de pessoas e orçamentos acima do previsto, pois a maioria desses novos modelos não apresentavam resultados prévios e se baseavam em teorias.

Depois desse período, Davenport (1990), com uma abordagem mais branda, sugeriu formas menos agressivas e mais inteligentes de aplicação nas metodologias de controle e gerenciamento de processos e passou a adotar a melhoria contínua dos processos de negócio e inovação. Nos anos seguintes, outros modelos de gestão, tais como gestão da qualidade total (TQM), os modelos de negócio e normas como a ISO 9000 tomaram um foco crescente em processos (STALK et al., 1992).

Contemporaneamente, o BPM é uma disciplina de gestão que exige das organizações uma mudança para o pensamento centrado nos processos e que ao longo dos últimos 15 anos tem recebido atenção crescente (DOEBELI et al., 2011). A abordagem é baseada em uma arquitetura de processos que captura as inter-relações entre os processos-chave de negócios, juntamente com a habilitação de processos de apoio e seu alinhamento com as estratégias, metas e políticas de uma organização (Armisted e Pritchard, 1999). O BPM está se tornando uma parte essencial das organizações contemporâneas. (PALMBERG, 2010).

A prática gerencial de BPM, de acordo com a ABPMP - Association of Business Process Management (2013), pode ser caracterizada como um ciclo de vida contínuo das atividades integradas: planejamento e estratégia, análise, desenho e modelagem, implantação, monitoramento e controle e refinamento. A figura 1 apresenta o detalhamento dessas atividades. 


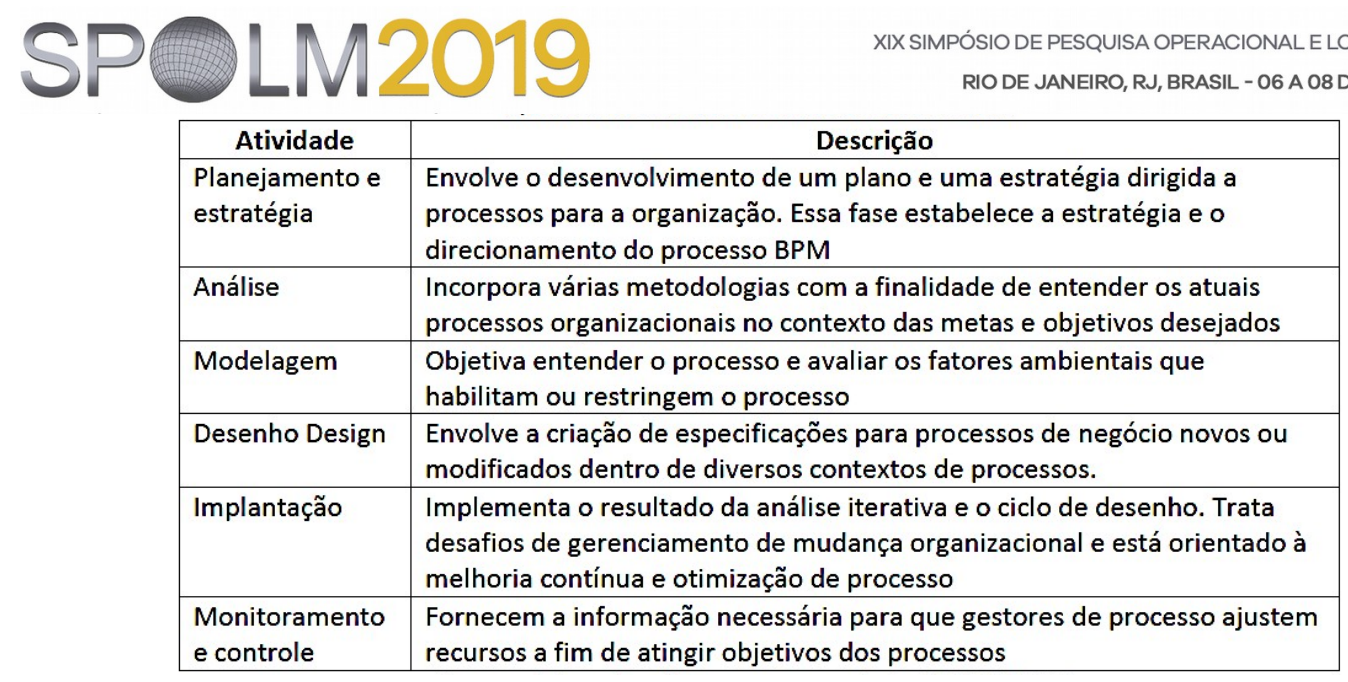

Figura 1: Atividades do ciclo de vida do BPM. Fonte: ABPMP (2013)

Conforme destaca Cruz (2000), usualmente as empresas possuem estruturas funcionais departamentalizadas, ou seja, as funções são distribuídas na forma de organogramas e podem, ou não, estar inter-relacionadas através das atividades que compõe um determinado processo. Nesta estrutura, a autoridade passa pelos níveis gerenciais para ser compartilhada com as funções técnicas e administrativas, dificultando a comunicação além de tornar lenta a reação às mudanças do mercado.

De acordo com Kipper et al. (2013), a gestão por processo permite que as organizações criem valores horizontais no processo. Redundando em atividades interrelacionadas tendo como fim último a valorização do cliente.

Os autores Jacobs et al. (2009) analisaram comparativamente, por meio de revisão bibliográfica, três metodologias de gestão por processos que, segundo os próprios autores, foram escolhidas por conta de suas semelhanças conceituais. São elas: Metodologia DOMP Documentação, Organização e Melhoria de Processo proposta por Cruz (2000), a metodologia Novo Olhar da Universidade de Santa Cruz do Sul (UNISC, 2010) e a metodologia GEPRO Gestão por Processos da Universidade Estadual de Campinas - UNICAMP de 2003.

Apesar das semelhanças encontradas entre as metodologias, Jacobs et al. (2009) destaca que a desenvolvida pela UNICAMP possui importantes etapas que precedem o mapeamento e redesenho, as quais são o planejamento estratégico, o entendimento do negócio, a análise dos processos críticos e a análise dos requisitos dos clientes e fornecedores.

\subsection{SISTEMAS INTEGRADOS DE GESTÃo}

Os sistemas integrados de gestão (SIG), o termo em inglês mais conhecido, Enterprise Resources Planning (ERP), entrega condições de suporte à procura de futuras oportunidades. Um dos objetivos principais da obtenção e implantação de um SIG é a possibilidade de um maior conhecimento e monitoração do desempenho da empresa, portanto, através disso, dando mais visibilidade as novas oportunidades do negócio e gerando mais eficiência operacional levando a uma vantagem competitiva (ESTEVES; JORGE, 2001).

Esteves e Jorge (2001), ressaltam que os sistemas integrados de gestão são pacotes de sistemas informatizados que têm como objetivo integrar diferentes áreas da empresa, ajudando e proporcionando recursos e processos aos usuários para uma gestão eficiente das informações, e obtendo, desta maneira, um excelente desempenho nas atividades operacionais e estratégicas, com o menor custo possível.

Alguns sistemas gerenciadores de informação são:

a) Sistemas de Planejamento dos Recursos Empresariais - Enterprise Resource Planning - ERP;

b) Sistemas de B.I. (Business Intelligence): englobam sistemas de informações gerenciais, sistemas de gestão de indicadores de desempenho (como, por exemplo, 
sistemas de Balanced Scorecard), data mining, data warehousing;

c) Sistemas de Automação de Processos: são sistemas como o workflow e sistemas de gestão eletrônica de documentos (GED);

d) Sistemas de Gestão do Relacionamento com Clientes: são sistemas como o CRM e o e-CRM, que auxiliam no processo de aquisição e retenção de clientes;

e) Sistemas de Gestão da Cadeia de Suprimentos: são sistemas como o SCM (Supply Chain Management) e o SRM (Supplier Relationship Management), que gerenciam os atores da cadeia de suprimentos e seu relacionamento;

f) Sistemas de Gestão do Conhecimento (Knowledge Management Systems - KMS): são sistemas que procuram reter o conhecimento explícito das organizações, além de gerenciar o conhecimento tácito.

Segundo, Seldin (2003), a obtenção de um sistema integrado ajuda no acompanhamento dos indicadores de desempenho da empresa, agiliza as estruturas de suporte para clientes e colaboradores da empresa, ajuda na identificação de problemas com produtos e sistemas de produção e na percepção de oportunidades e ameaças e por fim facilita na integração da cadeia de suprimentos e no relacionamento com fornecedores, parceiros, clientes assim como toda rede de stakeholders no geral.

É importante ressaltar, entretanto, que a implantação de um sistema integrado de gestão requer planejamento e um grande volume de investimento, além de uma equipe qualificada e treinada, com equipamentos necessários, tempo e recursos necessários para se alcançar os resultados do projeto de implantação. O cuidado em identificar pontos críticos na valorização de fatores subjetivos na Gestão de projetos de implantação de um ERP assume importantes posições no estabelecimento dos relacionamentos verticais entre as hierarquias da empresa. Tudo isso, ameniza e evita riscos que pode prejudicar o sucesso de um projeto de implantação de ERP. Dessa forma, é importante identificar através de lições aprendidas os benefícios e problemas de um Sistema ERP. A figura 2 mostra os benefícios e os problemas dos Sistemas ERP, dessa forma o quadro apresenta uma abordagem mais completa e analítica sobre a questão. 


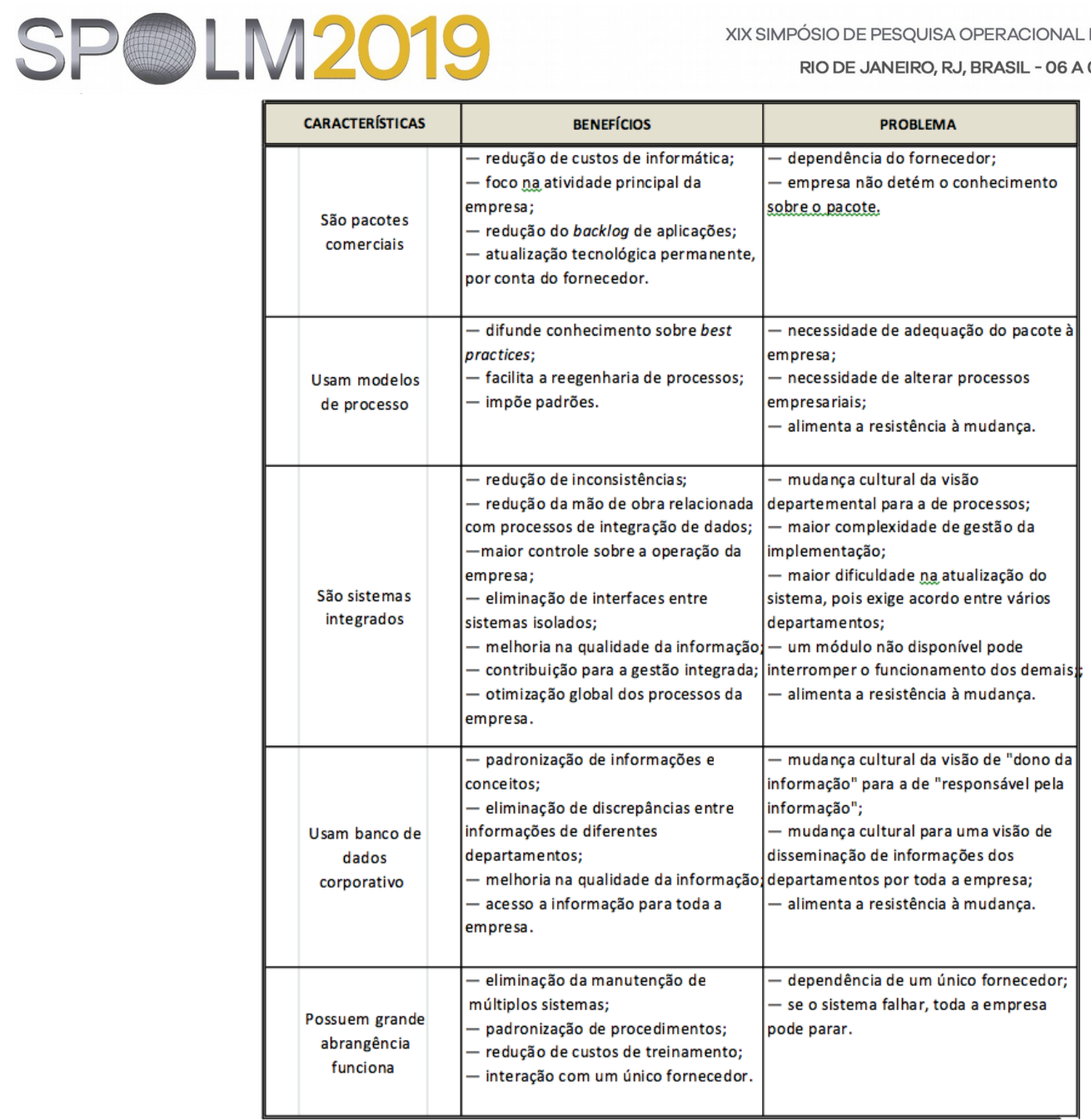

Figura 2: Benefícios e Problemas dos Sistemas ERP. Fonte: Souza e Zwicker (1999).

Segundo Seldin (2003), estes são alguns exemplos de pacotes comerciais de sistemas integrados de gestão são: Baan4 da holandesa Baan; EMS da brasileira Datasul/TOTVS; One World da americana JD Edwards; Oracle da americana Oracle; Peoplesoft da americana Peoplesoft e R/3 da alemã SAP.

\subsubsection{O MÉTODO ASAP NA IMPLANTAÇÃo DE UM ERP}

Segundo Esteves e Jorge (2001), existe uma metodologia específica de Gestão de Projetos na implantação de um sistema ERP SAP. Essa metodologia não exclui a utilização do guia PMBoK, pelo contrário, a metodologia ASAP serve como complemento para utilização dos gerentes de projetos para o sucesso na implantação do SAP.

A cultura da empresa se torna outra, depois de um projeto de implantação de ERP. É preciso se atentar com prazos, qualidade e custo, também é necessário que se realize uma gestão de conflitos e principalmente gestão da mudança. Uma empresa que tem uma cultura de empresa setorizada tem dificuldades de trabalhar de atuar de forma integrada, onde o produto gerado por um setor é dependente do término do serviço de outro para ser concluído. A empresa após a implantação do ERP tende a ficar mais integrada, as grandes empresas planejam um ERP para gerenciar a suas informações com o objetivo de melhora a tomada de decisões, porém se a empresa alcançar maturidade corporativa não obterá sucesso na automatização dos seus processos aumentando as chances de fracasso do projeto. O sucesso de uma implantação de ERP da forma correta faz com que a empresa se torne muito mais competitiva no mercado e o retorno sobre o investimento seja mais rápido que o esperado. No caso de uma implementação fraca, realizada de forma errada, o retorno sobre o investimento pode não surgir, sendo possível que acumule prejuízos (FORRESTER RESEARCH, 2014). 
A Figura 3 (abaixo) apresenta de forma visual e simples a metodologia ASAP, usada em projetos de implantação do SAP.

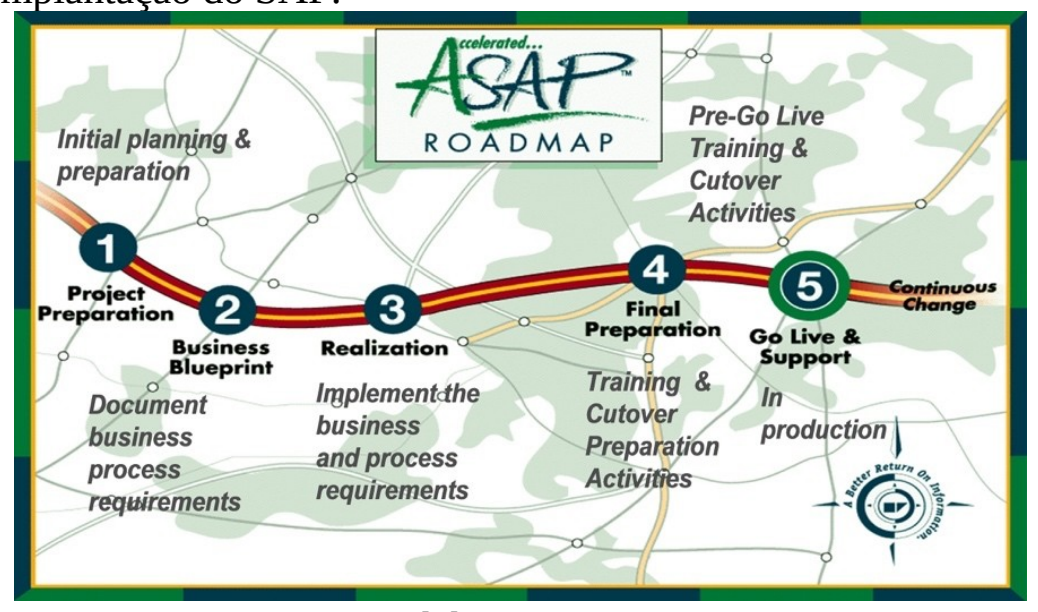

Figura 3: Metodologia ASAP. Fonte: Site 1.

Segundo o Site 1, a metodologia ASAP é dividida da seguinte forma:

1 - Project Preparation (preparação do projeto): é o inicio do projeto. Nele são definidos os key-users (usuários chave) e é planejada toda a definição estratégica do sistema.

Neste processo as definições a seguir são seguidas:

a) Definição do escopo de implementação;

b) Definição da estratégia de implementação;

c) Definição da organização e padrão de documentos;

d) Definição do cronograma da implementação e

e) Treinamento da equipe de projeto quanto ao método de implantação.

2 - Business Blueprint (modelo dos processos de negócio): gerar um documento denominado Business BluePrint, contendo os cenários, processos e requisitos de negócio.

Neste processo as definições a seguir são seguidas:

a) Definição da estrutura organizacional da empresa;

b) Desenho dos processos;

c) Revisão dos processos de negócio;

d) Documentação do escopo/desenho dos processos e

e) Treinamento da equipe de projeto nos processos abordados.

3 - Realization (realização): execução do Business Blueprint.

Neste processo as definições a seguir são seguidas:

a) Parametrização do sistema e da estrutura organizacional;

b) Desenvolvimento e Customização dos processos baseados no Business Blueprint.

c) Criação dos perfis de autorização e

d) Testes integrados.

4 - Final Preparation (preparação final): analisar e preparar o projeto para o Go Live.

Nesta fase as tarefas principais são:

a) Plano de entrada em produção;

b) Teste da carga de dados/Volume de dados e

c) Treinamento dos usuários finais.

5 - Go Live (entrada em produção): entrada em produção do sistema.

A utilização de sistemas ERP facilita o acesso a mapas gerenciais complexos, visão estratégica da empresa, união de setores, otimização nos processos e conduz a empresa a uma estrutura competitiva no mercado, utilizando de melhores práticas nos processos 
implementados. O desempenho da empresa se torna mais ágil, ajudando a empresa a tomar as decisões com base em dados reais.

\section{DISCUSSÃO DO TEMA E RESULTADOS}

O projeto pode atender uma necessidade temporária e trazer resultados significativos para a organização. Porém, por mais que aconteça um ciclo de melhorias geradas pelos projetos é essencial que a gestão de processos seja aprimorada. Dessa forma, a gestão de projetos pode conduzir excelentes resultados de curto e médio prazo, porém a empresa só conseguirá manter e aprimorar esses êxitos se gerenciar bem os seus processos, por isso entender a relação entre essas duas práticas de gestão é essencial. A compreensão entre a relação, interfaces, entendimento da definição de papéis da gestão de projetos e gestão de processos para a estratégia e planejamento da empresa geram resultados que agregam valor a empresa, conforme pode ser visto na figura 4 a seguir.

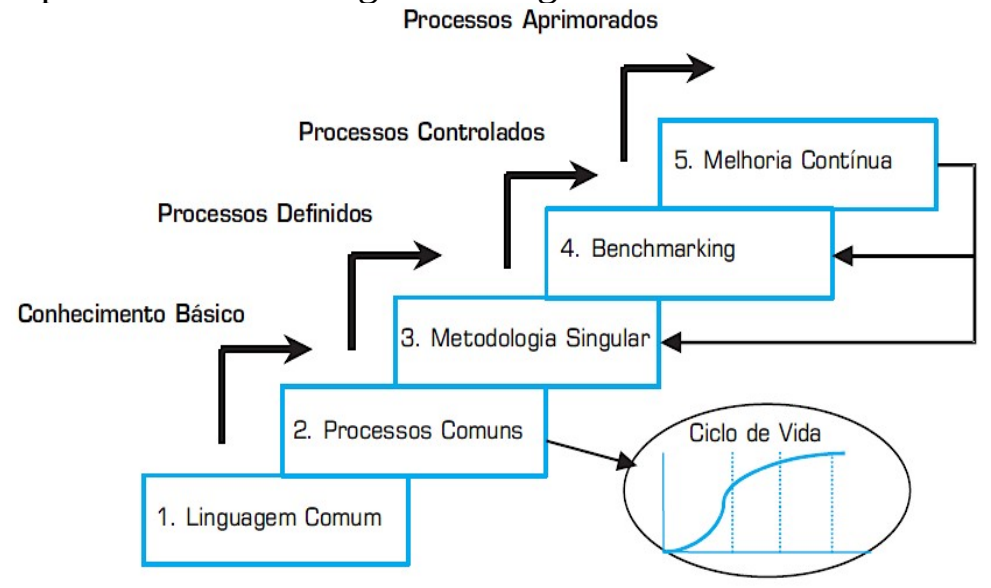

Figura 4: Project Management Maturity Model - PMMM. Fonte: Kerzner (2003).

No caso do objeto do estudo, falando da interface da Gestão de Processos e Gestão de Projetos na implantação de um ERP (SAP), esse desenvolvimento da maturidade nos processos ocorre em diversos níveis. É importante ressaltar que essa maturidade processual só ocorre depois que a Gestão do Projeto é realizada.

\subsection{Business Blue Print: A Interseção entre Gestão de Projetos e Processos}

Em se tratando de implantação do SAP, com a utilização da metodologia de Gestão de Projetos ASAP, a Gestão de Processos, durante a execução do projeto, é inserida mais diretamente na etapa de BBP - Business Blue Print -, onde são desenhados e definidos todos os processos. Dessa forma, as técnicas de Gestão de Processos são inseridas diretamente no projeto, para que a implantação tenha os resultados esperados. É importante ressaltar que a etapa de BBP é elementar para o sucesso do projeto, podemos considerar como o pilar do desenvolvimento de todas as outras etapas, pois a partir do que for definido nessa etapa definirá tudo que será feito nas etapas a frente.

Pode-se afirmar que a etapa de BBP é o ápice do ponto de encontro entre as duas ferramentas de Gestão que são objetos de estudo. Pois, a interface das técnicas de Gestão de Projetos e Gestão de Processos são usadas mais intensamente nessa etapa.

O Gerente de Projetos, precisa alinhar as competências em Gestão de Projetos e Gestão de Processos necessárias e utilizá-las da melhor forma para que um projeto de implantação de ERP atinja os resultados esperados através da metodologia ASAP.

Essa reformulação dos processos não ocorre somente a nível sistêmico, mas também a nível organizacional. Isso, portanto, reforça o papel da Gestão da Mudança nesse modelo de 
projeto, com o objetivo de avaliar os impactos das mudanças, atuando sobre eles, para que o a implantação do ERP obtenha o sucesso esperado e os retornos planejados.

\subsection{Implantação do SAP: Projeto Gerador de Melhorias Processuais}

A implantação do SAP não traz melhorias sistêmicas, mas melhorias processuais. É importante ressaltar que essas mudanças geradas pela implantação do SAP são a nível organizacional, exigindo intensa atuação das áreas de negócios, principalmente na fase de Business Blue Print. Fica a cargo das áreas de negócios as principais definições do desenho no novo sistema e das mudanças advindas desse projeto. Assim também como essas áreas de negócios serão as principais beneficiárias das facilidades advindas do SAP. Apesar de exigir alto grau de atuação do setor de Tecnologia da Informação num projeto de implantação de SAP, a principal função dele é de suporte, cabendo as funções de decisões estratégicas e processuais as áreas de negócios. O setor de tecnologia da informação, portanto é dependente das decisões tomadas pelas áreas de negócios.

Segundo Seldin (2003), a implantação de um sistema ERP SAP em uma empresa é um projeto que gera inúmeras melhorias a diversos níveis processuais. Após o término da implantação, após Go Live, é, portanto, onde ocorrem os principais benefícios da interface entre a Gestão de Projetos e Gestão de Processos. Dessa forma, pode-se ver que os benefícios de uma Gestão de Projetos bem executada geram processos melhores para a empresa levando a um maior nível de maturidade corporativa, facilitando a tomada de decisões da empresa através de maior integração entre os setores e melhor gerenciamento das informações proporcionadas por uma implantação realizada com o sucesso desejado. A figura 5 demonstra como a gestão de projetos pode servir como ferramenta para melhorias e restruturações processuais.

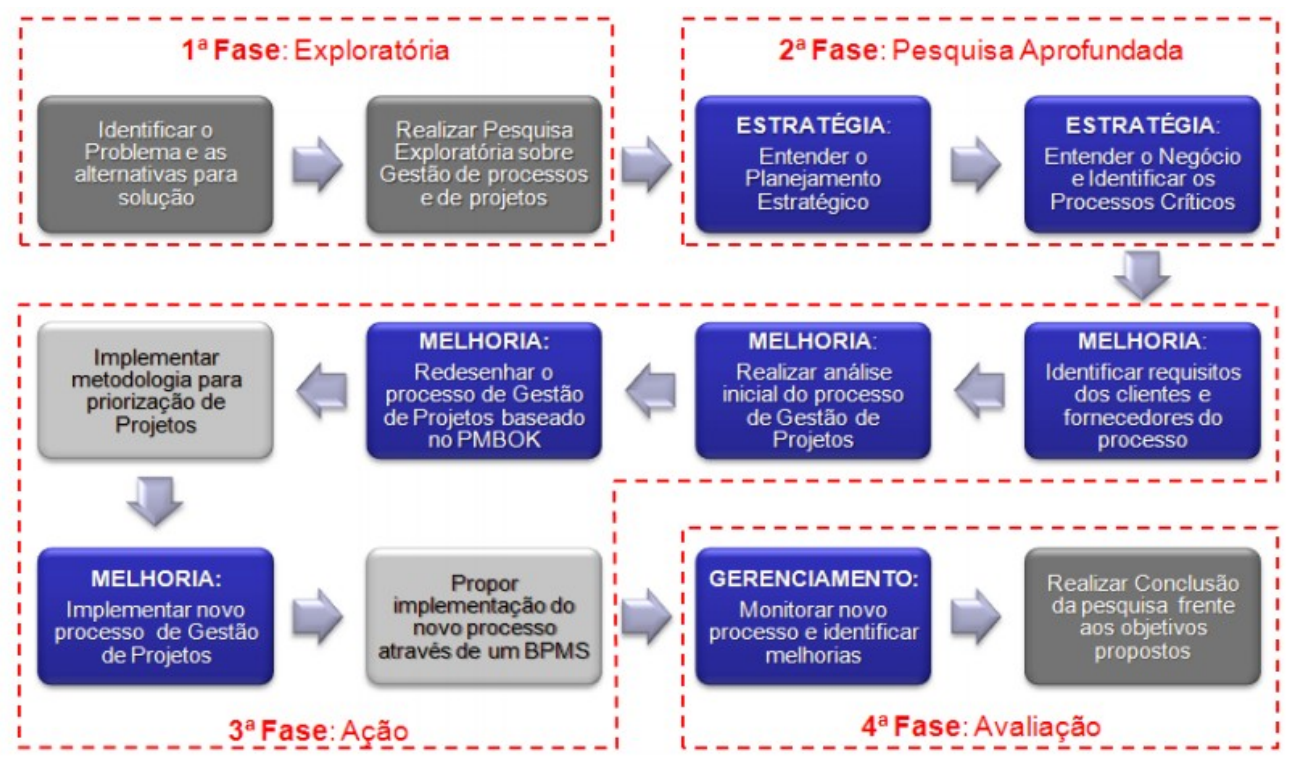

Figura 5: Gestão de Projetos como melhoria de processos. Fonte: Kipper et al. (2013)

É importante ressaltar que para que a Gestão do Projeto alcance os resultados esperados é necessário que a fase de BBP seja realizada da melhor forma possível e para que isso ocorra as ferramentas de Gestão de Processos devem ser bem empregadas nessa etapa. E se o projeto de implantação de ERP do SAP obtém os resultados planejados, ele trará mudanças e melhorias significativas para os processos da empresa após o Go Live. Dessa forma, é clara que a ligação direta entre Gestão de Projetos e Gestão de Processos durante e depois da implantação de um sistema ERP SAP. 


\subsubsection{A Importância da Gestão de Projetos e Processo Após o Go Live}

Após o término da implementação, a utilização do sistema passa a fazer parte do diaa-dia das operações. Orlikovski e Hofman (1997) realizaram um estudo sobre a introdução de novas tecnologias e relatam a dificuldade em conhecer de antemão todas as suas possibilidades de uso. Este conhecimento só se daria após certo tempo de uso continuado da tecnologia, através de ideias que surgiriam durante o processo de utilização. Esta é uma consideração importante para a etapa de utilização de sistemas ERP, pois geralmente não se conhecem todas as possibilidades de uso no momento da implementação, quando grande parte do esforço é utilizada para fazer combinar o pacote com a organização. Somente após esta etapa é possível vislumbrar novas alternativas e possibilidades de uso na empresa.

Desta maneira, a etapa de atualização realimenta a etapa de implementação com novas necessidades que possivelmente serão atendidas por outros módulos e com "condições de contorno", isto é, parâmetros do sistema já estabelecidos e em uso que só poderão ser alterados mediante nova mudança em procedimentos operacionais.

A Deloitte Consulting (1998), apresentando os resultados de uma pesquisa realizada em agosto de 1998 com 64 empresas que já implementaram sistemas ERP e encontram-se na fase de utilização, mostra que muitos benefícios obtidos pelas empresas só foram percebidos algum tempo após o início das operações. Segundo a pesquisa, o início da operação do sistema (go live) é geralmente o único objetivo, ou benefício, atingido após a implementação. Os demais benefícios são obtidos em etapas sucessivas, no que a pesquisa chama de "segunda onda" (second wave) dos sistemas ERP, à medida que a empresa começa a perceber todas as potencialidades da utilização dos sistemas.

Segundo Davenport (1990), a implementação de sistemas ERP tem sido tratada como um projeto na maioria das empresas, ou seja, tem início, meio e fim. Mas está se percebendo que um projeto ERP não é um projeto, mas “um meio de vida”. O autor afirma que para obter os benefícios desejados dos sistemas ERP é preciso encará-los dessa maneira, e tomar as medidas gerenciais necessárias, tais como alocação de recursos para um centro permanente de adaptação do sistema ERP às novas necessidades.

Segundo a Deloitte Consulting (1998), os benefícios dos sistemas ERP só podem ser obtidos na etapa de utilização se após a implementação a empresa mantiver o foco e esforços na obtenção dos resultados.

Desse modo, para que as novas mudanças necessárias para a etapa, posterior a implantação, ocorram, serão necessários novos projetos, geridos da mesma maneira que a primeira implantação, porém em dimensões menores, mas com o mesmo foco. A interface entre a Gestão de Projetos e Gestão de Processos necessita ser contínua após o Go Live, para que as melhorias sejam contínuas e com isso a maturidade corporativa também seja elevada posteriormente ao Go Live.

\subsubsection{Benefícios da Integração de Gestão de Projetos e Processos na Implantação do SAP}

Segundo Murphy (2002), os benefícios da implementação de sistemas de informação gerenciais podem ser divididos em tangíveis e intangíveis. Os tangíveis podem ser definidos como aqueles que afetam diretamente os resultados da empresa, tais como redução de custo e geração de lucros. Os intangíveis são os que causam melhorias de desempenho do negócio, mas não afetam diretamente no resultado da empresa, tais como informações gerenciais, segurança etc. Os benefícios do uso de TI podem ser definidos como custo, produtividade, flexibilidade, qualidade e inovação, e esses benefícios podem ser entendidos como a oferta que a tecnologia traz para as organizações.

Um ERP possui diversas vantagens. A principal dela é integrar todos os 
departamentos e processos de uma empresa em um único sistema atendendo às necessidades particulares destes departamentos. Um projeto de implantação de ERP SAP é uma ferramenta que melhora a integração entre as áreas, solucionando um problema organizacional advindo de falhas processuais.

Dentre as vantagens da implementação do SAP, destacam-se a qualidade e clareza das informações geradas, as quais auxiliam de forma eficaz a tomada de decisão; maior agilidade nos processos e; integração de todos os setores, evitando o retrabalho.

\subsection{Proposta de Plano de Gerenciamento de Projetos na Implantação de um ERP}

Para que o trabalho realizado tenha uma utilidade prática no futuro, foram desenvolvidas ferramentas de Gestão de Projetos, baseadas em uma implantação de um ERP (SAP) em uma empresa. Essa proposta é baseada no que foi assimilada no presente trabalho.

Para isso, foram construídas TAP (Termo de Abertura do Projeto), EAP (Estrutura Analítica do Projeto), Lista de Requisitos, Cronograma e Matriz de Stakeholders.

Dessa forma, o trabalho pretende apresentar uma solução sobre o que foi apresentado através do Gerenciamento de Projetos, para conseguir alcançar os resultados desejados. Com o objetivo de propor uma solução prática e clara, essas ferramentas de Gerenciamento de Projetos que foram utilizadas, ajudam a facilitar o alcance de resultados, realizando a ponte entre a teoria apresentada pelo trabalho e uma solução prática através do Gerenciamento de Projetos, baseado no guia do PMI (2013).

A TAP, segundo o PMI (2013), é o documento que autoriza formalmente um projeto. Ele concede ao gerente de projetos a autoridade para aplicar os recursos organizacionais nas atividades do Projeto. O Gerente de Projetos sempre deve ser designado antes do início do planejamento e, de preferência, no desenvolvimento do termo de abertura.

Através do TAP elaborado, traçou-se como objetivo Esse Projeto objetiva reduzir custos operacionais logísticos e de distribuição, trazer confiabilidade financeira através de dados mais seguros, alcançar ganhos de produtividade, padronização e definição de processos claros com aumento da margem de lucro. Foram definidas Premissas, Restrições e Riscos através desse documento.

As Premissas foram: implantação dos módulos MM, FI e SD do SAP; apenas consultores sêniores envolvidos no projeto; implantação do SAP realizada apenas na matriz da empresa, não se estendendo para filiais. A Restrição foi: tempo máximo do projeto de 9 meses. E os Riscos foram: uma correta migração dos dados e período de testes ser muito curto. Além disso, foram construídos objetivos, marcos e papel de cada função na equipe do projeto proposto.

Quanto aos requisitos, segundo o PMI (2013), é o processo de definir e documentar as necessidades das partes interessadas para atingir os objetivos dos projetos. Os requisitos devem ser obtidos, analisados e registrados em detalhes suficientes para serem medidos durante a execução do projeto e serão a base para construção da EAP. Assim, os requisitos foram:

a) Orçamento

b) Prazo

c) Planejamento e Controle

d) Finanças

e) Recursos humanos

f) Contratos

g) Sistemas

A EAP (Estrutura Analítica do Projeto), segundo o PMI (2013), é um processo de subdivisão das entregas e do trabalho do projeto em componentes menores e mais facilmente gerenciáveis. Na EAP do Projeto proposto foram construídos os seguintes pacotes de trabalho: 
1. Gerência de Projetos

2. Preparar Projeto

3. Definir escopo de implementação.

4. Definir estratégia de implementação

5. Definir organização e padrão de documentos.

6. Definir cronograma da implementação

7. Definir Escopo /Desenho de processos

8. Analise de Requisitos

9. Definir modelo dos processos de Negócio Business Blueprint

10. Definir estrutura organizacional da empresa;

11. Desenhar processos;

12. Revisar processos de negócio;

13. Revisar Documentação do escopo/desenho dos processos;

14. Treinar equipe de projeto nos processos abordados.

15. Adequação e Construção de Módulos

16. Parametrizar sistema e estrutura organizacional;

17. Desenvolver e Customizar

18. Criar perfis de autorização

19. Validação/Testes

20. Testes integrados

21. Final Preparation

22. Planejar entrada de produção

23. Treinar Usuários Finais

24. Go Live - Entrada em Produção

O cronograma do Projeto de implantação de ERP proposto tem uma duração de 9 meses e utilizam como base a metodologia ASAP apresentada nos capítulos anteriores.

Segundo o PMI (2013), a matriz de stakeholders engloba todas as pessoas que de alguma forma podem influir no sucesso do projeto. Assim considera-se interessado desde o patrocinador, os fornecedores, os membros da equipe de projeto, os membros da diretoria da empresa e o público externo (usuários e vizinhos) que seja afetado pelo projeto. Eles foram divididos entre stakeholders primários e secundários.

Stakeholders primários:

1. Gerente de projeto

2. Patrocinador

3. Key Users

4. Consultores SAP

5. Equipe do Projeto

6. Usuários Finais

Stakeholders secundários:

1. Fornecedores

2. Administrativo para apoio logístico dos consultores (Hotel, transporte entre outros)

3. Área de compras

4. Área jurídica

Simulando através das ferramentas de Gerenciamento de Projetos as possibilidades reais e práticas do que já foi apresentado na teoria.

\subsection{ANÁlise de Resultados}

Temas de difícil acesso a pesquisas e como pouco assunto disponível. Não existiam tantas pesquisas acadêmicas e teóricas sobre o assunto. Apesar de ser um assunto muito em 
voga no mercado e ser considerável o número de projetos e o volume de investimento gasto com esse viés, existe muito pouco estudo sobre o assunto. Esse é um tipo de tema que tem ficado mais na prática do dia-a-dia das empresas e as mesmas não costumam ter boa gestão de conhecimento sobre lições aprendidas do assunto em questão.

Portanto, apesar de ser um assunto bastante discutido nas empresas, ainda é pouco desenvolvido no meio acadêmico, podendo ser muito melhor explorado por estudiosos da área. Visando através dessas pesquisas atender a alta demanda das empresas sobre a temática abordada.

Apesar de existirem muitos artigos que abordam Gestão de Processos, Gestão de Projetos e ERP, existem muito poucos que abordam os temas em conjunto. A maioria deles aborda em separado e apenas deixa ressalvas que podem ser utilizadas como bases para desenvolver estudos.

O trabalho é muito importante, na medida que existe uma grande demanda sobre o assunto e pouca pesquisa aprofundada e detalhada sobre o tema.

Mesmo projetos bem-sucedidos enfrentam problemas no seu decorrer. Sejam eles motivados pela cultura da empresa, por mau planejamento ou briga de egos, os percalços acabam custando caro para as companhias. Afinal, no mundo dos negócios, tempo é dinheiro e sistema que não funciona é igual a processos que não funcionam. Apontar uma razão principal é difícil, mas, existem alguns fatores comuns encontrados nas empresas que, fatalmente, levam ao mau resultado. O primeiro não tem nada a ver com questões técnicas, mas sim com a cultura das organizações.

Entretanto foi possível perceber através do conteúdo levantado que os Projetos de Implantação de ERP estão fadados a apresentar os seguintes problemas abaixo segundo o Site 2, senão apresentarem uma estrutura bem relacionada entre Gestão de Processos e Gestão de Projetos como esse trabalho propõe:

1. Falta de uma camada de gerenciamento de projetos: no mínimo, a empresas precisa conhecer as melhores práticas de gerenciamento descritas no guia PMBOK $5^{\circ}$ Edição, principal publicação feita pelo PMI (2013).

2. Falha no planejamento do projeto: essa talvez seja a fase mais crítica de um projeto. As empresas não podem deixar de escrever, fazer diagramas, relatórios que gerem conhecimento e lições aprendidas.

3. Processos críticos de negócios mal definidos: quase uma consequência do mau planejamento. Caso isso aconteça, a empresa terá de fazer mudanças no sistema depois de estar pronto.

4. Falha em detalhar os processos nas pontas: caso a empresa não conheça exatamente a rotina das pessoas que vão, de fato, utilizar o sistema, fatalmente fará algo inútil ou complicado demais.

5. Falta de envolvimento do empregado das pontas.

6. Falha em preparar o sistema para aguentar os picos de utilização: nenhum sistema é utilizado com a mesma frequência o tempo inteiro. É preciso saber o quanto ele aguenta e quanto terá de aguentar, quando for exigido em carga máxima.

7. Fidelizar os patrocinadores do projeto. Todos os envolvidos no projeto precisam ter consciência do que está no papel e saber que é isso que será realizado.

8. Iniciar a implantação antes de definir o escopo: nada acontece antes que o cronograma e os recursos estejam bem definidos e formalmente aprovados.

9. Ultrapassar o planejado pelo escopo: estratégias e cenários econômicos mudam, mas não é sempre possível modificar o projeto a cada novidade de mercado. Por isso, é fundamental ter um sistema bem definido de gerenciamento de mudanças.

10. Grandes modificações de software padrão: um sistema SAP tem muitas funções. Antes 
de modificar o software, certifique-se, realmente, que isso é necessário.

11. Falhas de testes: de $20 \%$ a $40 \%$ (site 1 ) do tempo total de projeto deve estar reservado para os testes. E eles só são válidos se forem devidamente documentados.

12. Falta de treinamento: é um erro reduzir o custo do projeto cortando o treinamento. É necessário ter um plano de treinamento, que serve, também, para avaliar o conhecimento dos usuários.

13. Falhas ao carregar os dados no sistema: um sistema ERP gera mudanças culturais na empresa. Muitas vezes, os funcionários estão acostumados a usar diversos sistemas legados, cada um referente a uma determinada época. Por isso é preciso definir o alcance do novo sistema. Falta de dados também é um problema. Se um usuário diz que precisa trabalhar com determinada informação, não significa, necessariamente, que ela exista.

14. Falha no cut over: a data de inauguração do novo sistema, e desligamento de antigo, deve estar definida e o processo planejado. É muito improvável fazer isso sem causar impacto. Este plano tem de ser discutido já na fase de planejamento do projeto.

15. Falhas após o go live: depois de estar tudo funcionando, não é difícil se deparar com um time de suporte mal dimensionado. Outros problemas são a falta de documentação e falhas no entendimento das responsabilidades dos envolvidos.

16. Deixar os testes para depois do go live: testes devem ser feitos durante a fase de testes. Testar quando o usuário está precisando da ferramenta tem grandes chances de apresentar problemas.

\section{CONSIDERAÇÕES FINAIS}

\subsection{CRÍticas E COMENTÁRIOS}

Os temas abordados nessa pesquisa, são de ampla utilização em empresas privadas, públicas e Organizações Sem Fins Lucrativos. Possuem alta demanda no mercado, além disso são quase sempre relacionados quando utilizados como ferramentas de implantação de ERP.

Porém, existem muito pouco material produzido e de qualidade para pesquisa e estudo acadêmico. A maioria das pesquisas relacionadas ao assunto que puderam ajudar na sustentação desse estudo foram baseadas em estudo de consultoria especializadas da área e do próprio SAP que possui uma grande quantidade de materiais disponíveis no seu site abordando o tema. Porém, artigos acadêmicos com viés teóricos são poucos.

Entretanto, não é difícil encontrar pesquisas de consultorias que deem base aos resultados gerados pela proposta dessa monografia. Isso facilitou a demonstração do que foi proposto. Não obstante, a fundamentação teórica baseou-se em artigos e livros acadêmicos.

\subsection{RECOMENDAÇÕES PARA FUTUROS TRABALHOS}

Recomenda-se este trabalho para a realização de pesquisas complementares de Gestão de Processos, Gestão da Mudança e implantação de ERP SAP R3. Procurando verificar conceitos, técnicas ou ferramentas de apoio que se adaptem às práticas do Gerenciamento de Projetos e seus nuances com os conceitos primeiramente abordados.

A pesquisa foi extensa e profunda, podendo servir de base de estudo para os principais temas abordados. Principalmente que existem poucos estudos e pesquisas acadêmicas voltadas para esse tema. Essa monografia poderá servir de base de apoio para todos estudos futuros que envolverem esse tema. Existe muita demanda do mercado para isso, em contrapartida a poucas pesquisas acadêmicas disponíveis que atendam a essa demanda. 


\section{REFERÊNCIAS}

\subsection{REFERÊNCIAS BIBLIOGRÁFICAS}

[1] ABPMP. Guia para o Gerenciamento de Processos do Negócio: Corpo Comum de Conhecimento. v. 3. Chicago: ABPMP, 2013

[2] BEINHOCKER, E.D. The Origin of Wealth Evolution, Complexity, and the Radical Remaking of Economics, Harvard Business School Press, Boston, 2007.

[3] CLEGG, B.;SHAW, D. Using process-oriented holonic (PrOH) modelling to increase understanding of information systems. Information Systems Journal. v 18, p. 447-47, 2008.

[4] CRUZ, T., Workflow - A Tecnologia que Vai Revolucionar os Processos. São Paulo: Atlas, 2000.

[5] DAVENPORT, Thomas H. The new industrial engineering: Information technology and business process redesign. Sloan Management Review, pp.11-27, 1990.

[6] DELOITTE CONSULTING. ERP's Second Wave: Maximizing the Value of ERP-Enabled Processes. Relatório de pesquisa publicado pela Deloitte Consulting, São Paulo: Deloitte, 1998.

[7] DOEBELI,G., FISHER, R., GAPP, R.; SANZOGNI, L. Using BPM governance to align systems and practice. Business Process Management Journal. v.17, p.184-202, USA, 2011.

[8] ESTEVES, J., JORGE, J., Análise Comparativa de Metodologias de Implantação de SAP. Portugal: Associação Portuguesa de Sistemas de Informação (APSI), 2001.

[9] FORREST RESEARCH. Um estudo Total Economic Impact da Forrester Encomendado pela SAP, Análise de Custo Projetado da Plataforma SAP HANA. Relatório de pesquisa publicado pela Forrest Research. São Paulo, 2014.

[10] GASNIER, D. G., Guia Prático para Gerenciamento de Projetos, 1 ed., IMAN. São Paulo, SP, 2000.

[11] HAMMER, M.; CHAMPY, J. Reengineering the Corporation: A Manifesto for Business Revolution. New York: Harper Collins, 1993.

[12] HARMON, P., WOLF, C. The state of business process management", BPTrends. USA, 2012.

[13] HELLSTRÖM, A. Conceptions of process management - an anlysis of the discourse in the management literature. 9th International QMOD Conference, Liverpool, p.8-10, 2006.

[14] JACOBS, G. et al. Por Onde Começar a Gestão por Processos? Uma Análise sobre Metodologias artigo apresentado no SIMPEP 2009: Simpósio de Engenharia de Produção, Bauru, São Paulo, SP, 09-11 de Novembro, 2009.

[15] KERZNER, H. Project Management: A systems to approach to planning, scheduling and controlling. New York: Wiley, 2003.

[16] KIPPER, L.M.; NARA, E.O.B; MENDES, F.I. Process Management Applied to Project Management: A Methodology for Project Management in Industry. Sistemas \& Gestão, v.8, n.4, 2013.

[17] MORONI, M. A.; Hansen, P. B. Gestão por Processos e a Gestão de Projetos: Um Modelo Gerencial para Alocação de Recursos, Gestão Industrial, Vol.2, No.1. São Paulo, 2006.

[18] MURPHY, T. Achieving business value from technology: a practical guide for today's executive. New Jersey: John Wiley \& Sons, 2002.

[19] ORLIKOVSKI, Wanda J.; Hofman, J. Debra (1997). An Improvisational Model for Change Management: The Case of Groupware Technologies. Sloan Management Review, p.11-21 USA, Winter, 1997.

[20] PALMBERG, K. Experiences of implementing process management: a multiple-case study, Business Process Management Journal, v.16, p.93 - 113, 2010.

[21] PMI, Um Guia do Conhecimento em Gerenciamento de Projetos (Guia PMBOK 5 Edição ) - São Paulo, 2013. 
[22] PSOMAS, E. L.; FOTOPOULOS, C. V.; KAFETZOPOULOS, D. P. Core process management practices, quality tools and quality improvement in ISO 9001 certified manufacturing companies. Business Process Management Journal, v.17, p.437 - 460, Atenas, 2011.

[23] SCHONBERGER, R. World Class Manufacturing: The Lessons of Simplicity Applied, USA, 1986.

[24] SELDIN, Renata, O Papel Da Cultura Organizacional Na Implantação De Sistemas Integrados De Gestão - Uma Abordagem Sobre Resistência A Mudanças, XXIII Encontro Nac. de Eng. de Produção - Ouro Preto, MG, Brasil, 21 a 24 de out, 2003.

[25] SOUZA, C., ZWICKER, R., Aspectos envolvidos na seleção e implementação de sistemas ERP. Anais da XXXIV Assembléia Anual do CLADEA, Porto Rico: 1999.

[26] STALK, G., EVANS, P. e SHULMAN, L. Competing on Capabilities: The New Rules of Corporate Strategy, Harvard Business Review, , p 57-69, Boston 1992.

[27] ULMER, J.; BELAUD, J.; LE LANN, J. Towards a pivotal-based approach for business process alignment. International Journal of Computer Integrated Manufacturing, v.24, p. 11, USA, 2011.

[28] UNISC - Universidade de Santa Cruz do Sul. , Normas para apresentação de trabalhos acadêmicos. 1. ed., EDUNISC, Santa Cruz do Sul, RS, 2010.

\subsection{REFERÊNCIAS ELETRÔNICAS}

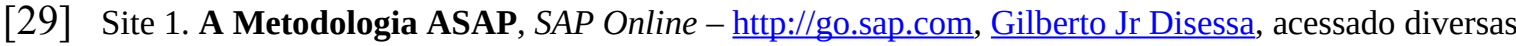
vezes entre março a junho de 2018.

[30] Site 2. Por que os Projetos de ERP Fracassam? http://computerworld.com.br/gestao/2009/08/18/porque-os-projetos-de-erp-fracassam , Rodrigo Caetano. Acessado em Fevereiro de 2019. 